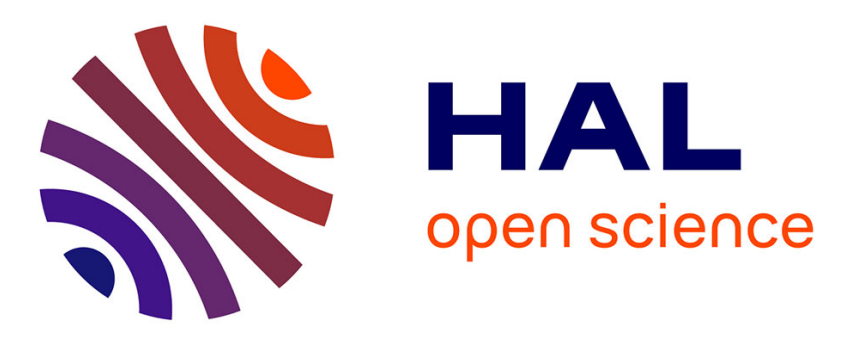

\title{
Super twisting algorithm based step-by-step sliding mode observers for nonlinear systems with unknown inputs
}

\author{
Thierry Floquet, Jean-Pierre Barbot
}

\section{To cite this version:}

Thierry Floquet, Jean-Pierre Barbot. Super twisting algorithm based step-by-step sliding mode observers for nonlinear systems with unknown inputs. International Journal of Systems Science, 2007, 38 (10), pp.803-815. 10.1080/00207720701409330 . inria-00128137v2

\section{HAL Id: inria-00128137 \\ https://hal.inria.fr/inria-00128137v2}

Submitted on 16 Feb 2007

HAL is a multi-disciplinary open access archive for the deposit and dissemination of scientific research documents, whether they are published or not. The documents may come from teaching and research institutions in France or abroad, or from public or private research centers.
L'archive ouverte pluridisciplinaire HAL, est destinée au dépôt et à la diffusion de documents scientifiques de niveau recherche, publiés ou non, émanant des établissements d'enseignement et de recherche français ou étrangers, des laboratoires publics ou privés. 


\title{
Super twisting algorithm based step-by-step sliding mode observers for nonlinear systems with unknown inputs
}

\author{
T. FLOQUET ${ }^{*}$ and J. P. BARBOT ${ }^{\ddagger}$
}

\begin{abstract}
This paper highlights the interest of step-by-step higher order sliding mode observers for MIMO nonlinear systems with unknown inputs. A structural matching condition, stating on the possibility to design such observers and to reconstruct the unknown inputs, is derived. A finite time sliding mode observer, based on the hierarchical use of the super twisting algorithm, is developed. Then, it is shown that this observer is of interest in the field of hybrid systems and systems with observability singularities. Lastly, it is shown through an example how to relax the usual matching condition by the means of this type of finite time sliding mode observer.
\end{abstract}

\section{Introduction}

The problem of designing an observer for a multivariable system partially driven by unknown inputs has been widely studied. Such observers can be of important use for systems subject to disturbances or with inaccessible inputs, and in many applications such as fault detection and identification or parameter identification. Many different approaches have been considered to design unknown input observers: conventional Luenberger design procedure under some decoupling and detectability conditions $[31,36,12]$ for linear systems, or high gain observers $[29,8,37]$ and algebraic methods [52] in the nonlinear case.

Other works rely on the use of discontinuous observers and sliding mode methodology (see [59, 21] or [48] for further information on sliding mode control and observation). The system trajectories are constrained to reach and stay, after a finite time, on a given sliding manifold for which the output error is zero. The sliding motion provides an estimation of the system state, asymptotically (see e.g. $[62,56,59,20,57,50,54]$ ) or in finite time (for instance, one can

\footnotetext{
* Corresponding author

$\dagger^{T}$. Floquet is with LAGIS, UMR CNRS 8146, Ecole Centrale de Lille, Cité scientifique, BP 48, 59651 Villeneuve d'Ascq Cedex, France, floquet@ec-lille.fr

$\ddagger$ J.P. Barbot are with ECS, ENSEA, 6 avenue du Ponceau, 95014 Cergy, FRANCE barbot@ensea.fr
} 
refer to the works of $[18,19,2,32,60]$ for design methods based on first order sliding mode algorithms and the works of $[41,4,53]$ for second order ones). Sliding mode observers have been shown to be a robust observation scheme in robotics $[9,35,45]$, electrical engineering $[15,60]$ or chemical reactors [46]. Specific applications of asymptotic and finite time observers for systems with unknown inputs can be found in a large number of fields such as fault detection and identification $[22,30,58,23]$, parameter identification [26, 55], cryptography [3], or mechanical systems with Coulomb friction [13, 27, 47].

The first part of this paper deals with the possibility to design finite time sliding mode observers for autonomous MIMO nonlinear systems subject to unknown inputs. Finite time sliding mode observers, are usually designed under the assumptions that the system can be put in a set of triangular observable forms, where the unknown inputs act only on the last dynamics of each triangular form. This assumption is known as the observability matching condition (it can be seen as the analogue of the well-known matching condition given in [17] for a sliding mode controller to be insensitive to matched perturbations). This condition was explicitly given in the case of SISO observable linear systems in [48], Chapter 4. Here, the condition is derived for MIMO nonlinear systems and is expressed in terms of the observability indices of the system and its relative degrees with respect to the unknown inputs. Then, a finite time higher order sliding mode observers, based on the iterative use of the super twisting algorithm (a second order sliding mode algorithm), is introduced in order to obtain the state and the unknown inputs in finite time.

The finite time convergence property of sliding mode observers is often desirable in the framework of observation and particularly for the purpose of observer-based controller design for nonlinear systems. Then, for a large class of nonlinear systems, the observer can be designed separately from the controller and the separation principle does not need to be proved. As shown in many papers, it can also be of paramount importance in applications that require fast estimations of some unknown inputs like fault detection and identification or on-line parameter identification. In the second part of this paper, other relevant applications are described. It is shown that finite time higher order sliding mode observers can cope with the problem of observability singularities that may occur in nonlinear systems. A straightforward application can be found in the domain of secure communication and cryptography, where the confidential information (the unknown inputs) can be hidden in observability bifurcations. Variable structure observers can also be of high interest to solve the observation problem of some class of hybrid systems (switched systems). Lastly, it is shown through an example that the matching conditions commonly assumed when designing sliding mode observers can be relaxed using the given sliding mode observer.

Notations: $L_{X} \psi$ denotes the classical Lie derivative of the function $\psi$ along the vector field $X$ and $d \psi$ is the differential of the function $\psi$. The codistribution $\Delta^{\perp}$ stands for the annihilator of the distribution $\Delta$. 


\section{Observability form and super twisting algo- rithm based step-by-step sliding mode observer}

Consider the following autonomous nonlinear system subject to unknown inputs:

$$
\left\{\begin{array}{l}
\dot{x}=f(x)+\sum_{i=1}^{m} g_{i}(x) w_{i} \\
y=h(x)=\left[h_{1}(x), . ., h_{p}(x)\right]^{T}
\end{array}\right.
$$

where $x \in U$, a neighborhood of $\mathcal{R}^{n}$, is the state vector, $y \in \mathcal{R}^{p}$ is the output vector and where $w=\left[w_{1}, \ldots, w_{m}\right] \in \mathcal{R}^{m}$ represents the unknown inputs. The vector fields $f$ and $g_{1}, \ldots, g_{m}$, and the functions $h_{1}, \ldots, h_{p}$ are assumed to be sufficiently smooth on $U$. It is assumed that $p \geq m$, and without loss of generality that, for all $x \in U$, the codistribution $\operatorname{span}\left\{d h_{1}, \ldots, d h_{p}\right\}$ is nonsingular. The system:

$$
\left\{\begin{array}{l}
\dot{x}=f(x) \\
y=h(x)
\end{array}\right.
$$

is supposed to be locally weakly observable (see [34] for a definition in the single output case). Hereafter, the definition given in [39] for the case of several outputs is recalled.

Definition 1 The system is locally weakly observable at $x^{0}$ if there exists $\mathcal{V}\left(x^{0}\right)$, a neighborhood of $x^{0}$, and $p$ integers $\left(k_{1}, k_{2}, \ldots, k_{p}\right)$ that form, after a possible reordering of the outputs, the smallest p-tuple with respect to the lexicographic ordering, such that:

(i) $k_{1} \geq k_{2} \geq \ldots \geq k_{p} \geq 0$

(ii)

$$
\sum_{i=1}^{p} k_{i}=n
$$

(ii)

$$
\operatorname{rank}\left(\begin{array}{c}
d h_{1}(x) \\
d L_{f} h_{1}(x) \\
\vdots \\
d L_{f}^{k_{1}-1} h_{1}(x) \\
\vdots \\
d h_{p} \\
\vdots \\
d L_{f}^{k_{p}-1} h_{p}(x)
\end{array}\right)=n
$$

for all $x \in \mathcal{V}\left(x^{0}\right)$.

The integers $\left(k_{1}, k_{2}, \ldots, k_{p}\right)$ are called the observability indices of (1). 


\subsection{Observability form}

Finite time sliding mode observers are usually designed under the assumption that the system can be put into a specific block triangular observable form where the unknown inputs appear in the last dynamics of each block only. Here, we give conditions for the MIMO nonlinear system (1) to be transformed into such a form. Let us first define the following integers $\left\{\rho_{1}, \ldots, \rho_{p}\right\}$ such that, for $i=1: p$ :

$$
\begin{aligned}
L_{g_{j}} L_{f}^{k} h_{i}(x) & =0, \text { for } k<\rho_{i}-1, \text { and for all } j=1: m, \\
L_{g_{j}} L_{f}^{\rho_{i}-1} h_{i}(x) & \neq 0, \text { for at least one } j=1: m,
\end{aligned}
$$

for all $x \in \mathcal{V}\left(x^{0}\right)$.

Proposition 1 Assume the system (1) is locally weakly observable. There exists a change of coordinates such that (1) is locally transformed into

$$
\begin{aligned}
\dot{\xi}^{i} & =A_{i} \xi^{i}+F_{i}(\xi)+G_{i}(\xi) w \\
y_{i} & =\left[\begin{array}{llll}
1 & 0 & \ldots & 0
\end{array}\right] \xi^{i}
\end{aligned}
$$

where

$$
\begin{aligned}
& \xi=\left[\begin{array}{lll}
\left(\xi^{1}\right)^{T} & \ldots & \left(\xi^{p}\right)^{T}
\end{array}\right]^{T}, \xi^{i} \in \mathcal{R}^{k_{i}} \\
& A_{i}=\left(\begin{array}{ccccc}
0 & 1 & 0 & 0 & 0 \\
0 & 0 & 1 & 0 & 0 \\
\vdots & \vdots & \vdots & \ddots & \vdots \\
0 & 0 & 0 & 0 & 1 \\
0 & 0 & 0 & 0 & 0
\end{array}\right) \in \mathcal{R}^{k_{i} \times k_{i}}, F_{i}(\xi)=\left(\begin{array}{c}
0 \\
\vdots \\
0 \\
L_{f}^{k_{i}} h_{i}(x)
\end{array}\right) \in \mathcal{R}^{k_{i}} \\
& G_{i}(\xi)=\left(\begin{array}{ccc}
0 & 0 & 0 \\
\vdots & \vdots & \vdots \\
0 & 0 & 0 \\
L_{g_{1}} L_{f}^{k_{i}-1} h_{i}(x) & \ldots & L_{g_{m}} L_{f}^{k_{i}-1} h_{i}(x)
\end{array}\right) \in \mathcal{R}^{k_{i} \times m}
\end{aligned}
$$

if and only if $k_{i} \leq \rho_{i}$ for all $i=1: p$ and for all $x \in \mathcal{V}\left(x^{0}\right)$.

Proof Since the system (1) is locally weakly observable, the following change of coordinates

$$
\xi=\phi(x)=\operatorname{col}\left\{h_{i}(x), \ldots, L_{f}^{k_{i}-1} h_{i}(x)\right\}_{i=1: p}
$$


is a local diffeomorphism. Since $k_{i} \leq \rho_{i}$ for all $i=1: p$, one has:

$$
\begin{aligned}
\frac{d h_{i}(x)}{d t}= & \frac{\partial h_{i}(x)}{\partial x}\left(f(x)+\sum_{j=1}^{m} g_{j}(x) w_{j}\right)=L_{f} h_{i}(x) \\
\frac{d\left(L_{f} h_{i}(x)\right)}{d t}= & \frac{\partial L_{f} h_{i}(x)}{\partial x}\left(f(x)+\sum_{j=1}^{m} g_{j}(x) w_{j}\right)=L_{f}^{2} h_{i}(x) \\
\frac{d\left(L_{f}^{k_{i}-1} h_{i}(x)\right)}{d t}= & L_{f}^{k_{i}} h_{i}(x)+\sum_{j=1}^{m} L_{g_{j}} L_{f}^{k_{i}-1} h_{i}(x) w_{j}
\end{aligned}
$$

Thus, the system is described by (2)-(3) in the new coordinates, with $G_{i}(\xi) \neq 0$ if and only if $k_{i}=\rho_{i}$.

As a consequence, the system (1), even if it is weakly observable, can not be put into the block triangular observable form (2)-(3) if $\sum_{i=1}^{p} \rho_{i}<n$.

Remark 1 The condition $k_{i} \leq \rho_{i}$ can also be stated in terms of codistribution. Indeed,

$$
k_{i} \leq \rho_{i} \Longleftrightarrow g_{j}(x) \in \Omega_{i}^{\perp}(x), \text { for all } x \in \mathcal{V}\left(x^{0}\right), \text { for all } j=1: m,
$$

where

$$
\Omega_{i}(x)=\operatorname{span}\left\{d h_{i}(x), d L_{f} h_{i}(x), \ldots, d L_{f}^{k_{i}-2} h_{i}(x)\right\} .
$$

Remark 2 For sake of simplicity, the case of nonlinear systems with known inputs is not discussed here. It is known that observability of nonlinear systems depends on known control inputs $u$. However, the result given in Proposition 1 can be extended for uniformly observable systems, i.e. systems which are observable independently of the inputs (see [28] for a work on uniformly observable systems and [2] for a sliding mode observer design for such systems).

Finite time sliding mode observers are usually designed for system in the form (2)-(3). One can refer to the works $[32,60]$ for linear and linear time varying systems or to $[18,19,2,63]^{1}$ for nonlinear systems. All of them are based on the analyze of the so-called equivalent output injection (which is the counterpart of the equivalent control in the design of sliding mode control). The estimation of the state is based on a procedure using the successive filtered values of equivalent output injections obtained from recursive first order sliding mode observers. However, the approximation of the equivalent output injections by low pass filters at each step may introduce some delays that could lead to inaccurate estimates or to instability for high order systems ${ }^{2}$. To overcome this

\footnotetext{
${ }^{1}$ Note that in [63], a first order sliding mode observer is designed for a system with asymptotically stable zero dynamics. This results in a partial finite time stabilization of the state.

${ }^{2}$ In [33], the implementation of such filters was studied and a relation between the estimation accuracy, the filter time constants and the sampling time was given.
} 
problem, a second order sliding mode algorithm can be used in the innovative term of the observer. In the next part, a super twisting based sliding mode observer is designed for the finite time estimation of a $n$-order system in the form (2)-(3).

\subsection{A finite time step-by-step sliding mode observer}

A robust and finite time exact differentiator based on the super twisting algorithm (a second order sliding mode algorithm [40]) was introduced in [41] and successfully applied in [27] and [49]. This approach was extended to the design of arbitrary order robust exact differentiators with finite time convergence in $[42,43]$ using homogeneity properties and in [6] using some hierarchical procedure. In [2], a so-called step-by-step first order sliding mode observer for the finite time estimation of the state variables was developed. Hereafter, a similar observer based on the super twisting algorithm is given.

\subsubsection{SISO case}

Let us consider a SISO nonlinear system in triangular observable form:

$$
\left\{\begin{array}{l}
\dot{x}_{1}=x_{2}, \ldots, \dot{x}_{n-1}=x_{n} \\
\dot{x}_{n}=\alpha(x)+\beta(x) \omega
\end{array}\right.
$$

where $x=\left[x_{1}, \cdots, x_{n}\right]^{T} \in \mathcal{R}^{n}$ is the state vector, $y=x_{1} \in \mathcal{R}$ is the output vector and $\omega \in \mathcal{R}$ is the unknown input. $\alpha(x)$ and $\beta(x)$ are bounded smooth scalar functions. Let us assume that the state of the system is uniformly bounded, i.e. for all $t>0,\left|x_{i}(t)\right|<d_{i}$, and that for all $t>0$ :

$$
\begin{aligned}
& |\omega(t)|<K_{1},|\dot{\omega}(t)|<K_{1}^{\prime}, \\
& |\alpha(t)|<K_{2},|\dot{\alpha}(t)|<K_{2}^{\prime}, \\
& |\beta(t)|<K_{3},|\dot{\beta}(t)|<K_{3}^{\prime},
\end{aligned}
$$


where $K_{i}$ and $K_{i}^{\prime}$ are some known positive scalars. Let us design the following observer:

$$
\left\{\begin{array}{l}
\dot{\hat{x}}_{1}=\tilde{x}_{2}+\lambda_{1}\left|e_{1}\right|^{1 / 2} \operatorname{sign}\left(e_{1}\right) \\
\dot{\tilde{x}}_{2}=\alpha_{1} \operatorname{sign}\left(e_{1}\right) \\
\dot{\hat{x}}_{2}=E_{1}\left[\tilde{x}_{3}+\lambda_{2}\left|e_{2}\right|^{1 / 2} \operatorname{sign}\left(e_{2}\right)\right] \\
\dot{\tilde{x}}_{3}=E_{1} \alpha_{2} \operatorname{sign}\left(e_{2}\right) \\
\dot{\hat{x}}_{3}=E_{2}\left[\tilde{x}_{4}+\lambda_{3}\left|e_{3}\right|^{1 / 2} \operatorname{sign}\left(e_{3}\right)\right] \\
\vdots \\
\dot{\tilde{x}}_{n-1}=E_{n-3} \alpha_{n-2} \operatorname{sign}\left(e_{n-2}\right) \\
\dot{\hat{x}}_{n-1}=E_{n-2}\left[\tilde{x}_{n}+\lambda_{n-1}\left|e_{n-1}\right|^{1 / 2} \operatorname{sign}\left(e_{n-1}\right)\right] \\
\dot{\tilde{x}}_{n}=E_{n-2} \alpha_{n-1} \operatorname{sign}\left(e_{n-1}\right) \\
\dot{\hat{x}}_{n}=E_{n-1}\left[\tilde{\theta}+\lambda_{n}\left|e_{n}\right|^{1 / 2} \operatorname{sign}\left(e_{n}\right)\right] \\
\dot{\tilde{\theta}}_{n}=E_{n-1} \alpha_{n} \operatorname{sign}\left(e_{n}\right)
\end{array}\right.
$$

where $e_{i}=\tilde{x}_{i}-\hat{x}_{i}$ for $i=1, . ., n$, with $\tilde{x}_{1}=x_{1}$ and $[\tilde{x}, \tilde{\theta}]^{T}=\left[\tilde{x}_{1}, \tilde{x}_{2}, \ldots \tilde{x}_{n}, \tilde{\theta}\right]^{T}$ is the output of the observer. For $i=1, \ldots, n-1$, the scalar functions $E_{i}$ are defined as

$$
E_{i}=1 \text { if }\left|e_{j}\right|=\left|\tilde{x}_{j}-\hat{x}_{j}\right| \leq \varepsilon, \text { for all } j \leq i \text { else } E_{i}=0 .
$$

where $\varepsilon$ is a small positive constant. The observer gains $\lambda_{i}$ and $\alpha_{i}$ are positive scalars.

The convergence of the state observation error is obtained in $(n-1)$ steps and in finite time.

First step: Assume $e_{1}(0) \neq 0$, the error dynamics is given by:

$$
\begin{aligned}
\dot{e}_{1} & =x_{2}-\tilde{x}_{2}-\lambda_{1}\left|e_{1}\right|^{1 / 2} \operatorname{sign}\left(e_{1}\right) \\
\dot{x}_{2} & =\alpha_{1} \operatorname{sign}\left(e_{1}\right) \\
\dot{e}_{2} & =\alpha_{1} \operatorname{sign}\left(e_{1}\right)-E_{1}\left[\tilde{x}_{3}+\lambda_{2}\left|e_{2}\right|^{1 / 2} \operatorname{sign}\left(e_{2}\right)\right] \\
\dot{e}_{i} & =E_{i-2} \alpha_{i-1} \operatorname{sign}\left(e_{i-1}\right)-E_{i-1}\left[\tilde{x}_{i+1}+\lambda_{i}\left|e_{i}\right|^{1 / 2} \operatorname{sign}\left(e_{i}\right)\right], \quad i=3, \ldots, n-1 \\
\dot{e}_{n} & =E_{n-2} \alpha_{n-1} \operatorname{sign}\left(e_{n-1}\right)-E_{n-1}\left[\tilde{\theta}+\lambda_{n}\left|e_{n}\right|^{1 / 2} \operatorname{sign}\left(e_{n}\right)\right]
\end{aligned}
$$

Equations (6)-(7) coincide with the so-called super twisting algorithm and the remaining dynamics is bounded in finite time. The second time derivative of $e_{1}$ is given by:

$$
\ddot{e}_{1}=x_{3}-\alpha_{1} \operatorname{sign}\left(e_{1}\right)-\frac{1}{2} \lambda_{1} \dot{e}_{1}\left|e_{1}\right|^{-\frac{1}{2}} .
$$

Sufficient conditions for the finite time convergence on the second order sliding 
set $\left\{e_{1}=\dot{e}_{1}=0\right\}$ are:

$$
\begin{aligned}
\alpha_{1} & >d_{3}, \\
\lambda_{1}^{2} & >4 d_{3} \frac{\alpha_{1}+d_{3}}{\alpha_{1}-d_{3}},
\end{aligned}
$$

and one can find an upper bound of the convergence time (see [41, 13, 53]). The equivalent dynamics provides after a finite time $T_{1}$ a continuous estimation of $x_{2}$ (without any introduction of low pass filters): $\tilde{x}_{2}=x_{2}$. The functions $E_{i}$ are introduced so that the $e_{i}$ 's, $i=2, \ldots, n$, do not escape two far before the good information on $\hat{x}_{1}$ and $\tilde{x}_{2}$ is obtained. Indeed,

$$
\begin{aligned}
\dot{e}_{2} & =\alpha_{1} \operatorname{sign}\left(e_{1}\right) \\
\dot{e}_{i} & =0, \quad i=3, \ldots, n
\end{aligned}
$$

except on a finite number of finite time intervals where $\left|e_{1}\right|$ may be less than $\varepsilon$ before the second order sliding set $\left\{e_{1}=\dot{e}_{1}=0\right\}$ is reached.

Second step: after a finite time, one has $E_{1}=1$ and the observer dynamics becomes:

$$
\begin{aligned}
\dot{e}_{1} & =0 \\
\dot{e}_{2} & =x_{3}-\tilde{x}_{3}-\lambda_{2}\left|e_{2}\right|^{1 / 2} \operatorname{sign}\left(e_{2}\right) \\
\dot{\tilde{x}}_{3} & =\alpha_{2} \operatorname{sign}\left(e_{2}\right) \\
\dot{e}_{3} & =\alpha_{2} \operatorname{sign}\left(e_{2}\right)-E_{2}\left[\tilde{x}_{4}+\lambda_{3}\left|e_{3}\right|^{1 / 2} \operatorname{sign}\left(e_{3}\right)\right] \\
\dot{e}_{i} & =E_{i-2} \alpha_{i-1} \operatorname{sign}\left(e_{i-1}\right)-E_{i-1}\left[\tilde{x}_{i+1}+\lambda_{i}\left|e_{i}\right|^{1 / 2} \operatorname{sign}\left(e_{i}\right)\right], i=4, \ldots, n-1 \\
\dot{e}_{n} & =E_{n-2} \alpha_{n-1} \operatorname{sign}\left(e_{n-1}\right)-E_{n-1}\left[\tilde{\theta}+\lambda_{n}\left|e_{n}\right|^{1 / 2} \operatorname{sign}\left(e_{n}\right)\right]
\end{aligned}
$$

Again, the structure of a super twisting algorithm appears. Thus, the trajectories converge in finite time onto $\left\{e_{2}=\dot{e}_{2}=0\right\}$. As a consequence, $\tilde{x}_{3}$ provides an estimation of $x_{3}$.

Following the same scheme till the $(n-1)$-th step, an estimation of the whole state $x$ is obtained in finite time. The finite time estimation of the unknown inputs can be obtained with the $n$-th step of the observer.

$\mathbf{n}$-th step: the observer dynamics is given by:

$$
\left\{\begin{array}{l}
\dot{e}_{1}=\ldots=\dot{e}_{n-1}=0 \\
\dot{e}_{n}=\alpha(x)+\beta(x) \omega-\tilde{\theta}-\lambda_{n}\left|e_{n}\right|^{1 / 2} \operatorname{sign}\left(e_{n}\right) \\
\dot{\tilde{\theta}}=\alpha_{n} \operatorname{sign}\left(e_{n}\right)
\end{array}\right.
$$

and a sliding motion appears after a finite time on the sliding manifold $e_{n}=$ $\dot{e}_{n}=0$. One has:

$$
\ddot{e}_{n}=\dot{\alpha}(x)+\dot{\beta}(x) \omega+\beta(x) \dot{\omega}-\alpha_{n} \operatorname{sign}\left(e_{n}\right)-\frac{1}{2} \lambda_{n} \dot{e}_{n}\left|e_{n}\right|^{-\frac{1}{2}} .
$$


Thus, choosing

$$
\begin{aligned}
& \alpha_{n}>K_{2}^{\prime}+K_{3}^{\prime} K_{1}+K_{3} K_{1}^{\prime}, \\
& \lambda_{n}^{2}>4\left(K_{2}^{\prime}+K_{3}^{\prime} K_{1}+K_{3} K_{1}^{\prime}\right) \frac{\alpha_{n}+K_{2}^{\prime}+K_{3}^{\prime} K_{1}+K_{3} K_{1}^{\prime}}{\alpha_{n}-\left(K_{2}^{\prime}+K_{3}^{\prime} K_{1}+K_{3} K_{1}^{\prime}\right)},
\end{aligned}
$$

a second order sliding motion appears on $\left\{e_{n}=\dot{e}_{n}=0\right\}$. Then, a continuous approximation $\tilde{\omega}$ of the unknown variables, on every subset of $\mathcal{R}^{n}$ where $\beta(x) \neq$ 0 , is:

$$
\tilde{\omega}=\beta^{-1}(\tilde{x})[\tilde{\theta}-\alpha(\tilde{x})] .
$$

The successive equivalent output injections on the sliding manifolds are obtained in a continuous way (they are all outputs of some integrators and so they are less affected by the chattering phenomenon) and without any introduction of low pass filters.

\subsubsection{MIMO case}

Consider a system with $p$ outputs and $m$ unknown inputs in the form (2)-(3). It is constituted of $p$ blocks in a triangular observable form similar to (4). The design of an observer (5) for each block results in the finite time estimation of the state variables and the following relations:

$$
L_{f}^{k_{i}} h_{i}(x)+\sum_{j=1}^{m} L_{g_{j}} L_{f}^{k_{i}-1} h_{i}(x) w_{j}=\tilde{\theta}_{i}, i=1: p
$$

where $\tilde{\theta}_{i}$ is the equivalent output injection of the last observer variable of each block. The relations (9) can be rewritten as:

$$
\left(\begin{array}{ccc}
L_{g_{1}} L_{f}^{k_{1}-1} h_{1}(x) & \ldots & L_{g_{m}} L_{f}^{k_{1}-1} h_{1}(x) \\
\vdots & & \vdots \\
L_{g_{1}} L_{f}^{k_{p}-1} h_{p}(x) & \ldots & L_{g_{m}} L_{f}^{k_{p}-1} h_{p}(x)
\end{array}\right) w=\left(\begin{array}{c}
\tilde{\theta}_{1}-L_{f}^{k_{1}} h_{1}(x) \\
\vdots \\
\tilde{\theta}_{p}-L_{f}^{k_{p}} h_{p}(x)
\end{array}\right)
$$

Let us assume that the distribution $\operatorname{span}\left\{g_{1}(x), \ldots, g_{m}(x)\right\}$ is nonsingular for all $x \in \mathcal{V}\left(x^{0}\right)$. Then, the matrix

$$
\Lambda(x)=\left(\begin{array}{ccc}
L_{g_{1}} L_{f}^{k_{1}-1} h_{1}(x) & \ldots & L_{g_{m}} L_{f}^{k_{1}-1} h_{1}(x) \\
\vdots & & \vdots \\
L_{g_{1}} L_{f}^{k_{p}-1} h_{p}(x) & \ldots & L_{g_{m}} L_{f}^{k_{p}-1} h_{p}(x)
\end{array}\right)
$$

has full column rank since:

$$
\Lambda(x)=\left(\begin{array}{c}
d L_{f}^{k_{1}-1} h_{1}(x) \\
\vdots \\
d L_{f}^{k_{p}-1} h_{p}(x)
\end{array}\right)\left(\begin{array}{lll}
g_{1}(x) & \ldots & g_{m}(x)
\end{array}\right)
$$


Thus, an estimation of the unknown input is given by:

$$
\tilde{w}=\Lambda^{+}(\tilde{x})\left(\begin{array}{c}
\tilde{\theta}_{1}-L_{f}^{k_{1}} h_{1}(\tilde{x}) \\
\vdots \\
\tilde{\theta}_{p}-L_{f}^{k_{p}} h_{p}(\tilde{x})
\end{array}\right)
$$

where $\Lambda^{+}$is a well defined pseudo-inverse of $\Lambda$.

Remark 3 In this case, the $p \times m$ matrix

$$
\Gamma(x)=\left(\begin{array}{ccc}
L_{g_{1}} L_{f}^{\rho_{1}-1} h_{1}(x) & \ldots & L_{g_{m}} L_{f}^{\rho_{1}-1} h_{1}(x) \\
\vdots & & \vdots \\
L_{g_{1}} L_{f}^{\rho_{p}-1} h_{p}(x) & \ldots & L_{g_{m}} L_{f}^{\rho_{p}-1} h_{p}(x)
\end{array}\right)
$$

has also full column rank. Then, the set of integers $\left\{\rho_{1}, \ldots, \rho_{p}\right\}$ is exactly the vector relative degree as it is defined in [38], p. 220.

\section{Some applications of higher order sliding mode observers}

\subsection{Variable structure observer and observability singu- larity}

Loss of observability may locally appear in nonlinear systems because of the nonlinear structure itself [3] or due to singular inputs (i.e. inputs that make the system unobservable, see [28]). This section highlights the efficiency of finite time sliding mode observers in the case of systems with local observability singularity. Roughly speaking, the variable structure of the sliding mode observer is very well suited to the change of structural properties like the transient between full state observability and partial state observability. This point is illustrated in the case of the following nonlinear system with an unknown input $m$ :

$$
\begin{aligned}
\left(\begin{array}{c}
\dot{x}_{1} \\
\dot{x}_{2} \\
\dot{x}_{3}
\end{array}\right) & =\left(\begin{array}{c}
x_{2} \\
-x_{1}+x_{2} x_{3} \\
x_{3}-\left(1+x_{1}^{2}+x_{2}^{2}\right) x_{3}^{3}+m
\end{array}\right):=f(x)+g(x) m \\
y & =x_{1}:=h(x)
\end{aligned}
$$

This system is locally weakly observable because the rank condition given in [34] is satisfied, i.e.:

$$
\operatorname{rank}\left(\begin{array}{c}
d h \\
d L_{f} h \\
d L_{f}^{2} h \\
d L_{f}^{3} h \\
\vdots
\end{array}\right)=\operatorname{rank}\left(\begin{array}{ccc}
1 & 0 & 0 \\
0 & 1 & 0 \\
-1 & x_{3} & x_{2} \\
* & * & -x_{1}+x_{2}\left(1+2 x_{3}-3 x_{3}^{2}\left(1+x_{1}^{2}+x_{2}^{2}\right)\right) \\
\vdots & \vdots & \vdots
\end{array}\right)=3
$$


Actually, for $x_{2}=0$, the observability of the system is obtained at least with the third time derivative of $y$. Nevertheless, (10) does not satisfy the observability matching condition given in Proposition 1 if $x_{2}=0$. This means that usual classical sliding mode observers ${ }^{3}$ can only be designed if the system trajectories always evolve outside the following manifold of observability singularity $\mathcal{S}=$ $\left\{x \in \mathcal{R}^{3}: x_{2}=0\right\}$. In the vicinity of $\mathcal{S}$, the observer must change of structure. Thus, the following modified finite time sliding mode observer is introduced in this paper:

$$
\left\{\begin{aligned}
\dot{\hat{x}}_{1}= & \tilde{x}_{2}+\lambda_{1}\left|\tilde{e}_{1}\right|^{1 / 2} \operatorname{sign}\left(\tilde{e}_{1}\right) \\
\dot{\tilde{x}}_{2}= & \alpha_{1} \operatorname{sign}\left(\tilde{e}_{1}\right) \\
\dot{\hat{x}}_{2}= & E_{1}\left[-x_{1}+v_{2}+\lambda_{2}\left|\tilde{e}_{2}\right|^{1 / 2} \operatorname{sign}\left(\tilde{e}_{2}\right)\right] \\
\dot{v}_{2}= & E_{1} \alpha_{2} \operatorname{sign}\left(\tilde{e}_{2}\right) \\
\dot{\hat{x}}_{3}= & E_{2}\left[\left(E_{s} \tilde{x}_{3}+\left(1-E_{s}\right) \hat{x}_{3}\right)-\left(1+x_{1}^{2}+\hat{x}_{2}^{2}\right)\left(E_{s} \tilde{x}_{3}^{3}+\left(1-E_{s}\right) \hat{x}_{3}^{3}\right)\right. \\
& \left.+K E_{s} \operatorname{sign}\left(\tilde{x}_{3}-\hat{x}_{3}\right)\right]
\end{aligned}\right.
$$

where $\tilde{e}_{i}=\tilde{x}_{i}-\hat{x}_{i}$, with $\tilde{x}_{1}=x_{1}(i=1,2,3) . \lambda_{1}, \alpha_{1}, \lambda_{2}, \alpha_{2}$, and $K$ are positive constant and the functions $E_{i}(i=1,2)$ and $E_{s}$ are given by:

$$
E_{i}=1 \text { if }\left|\tilde{e}_{j}\right|=\left|\tilde{x}_{j}-\hat{x}_{j}\right| \leq \varepsilon \text { for all } j \leq i, \text { else } E_{i}=0
$$

and

$$
E_{s}=0 \text { if }\left|\tilde{x}_{2}\right| \leq \varepsilon, \quad \text { else } \quad E_{s}=1
$$

$\tilde{x}_{3}$ is defined as:

$$
\tilde{x}_{3}=E_{1} E_{s} \frac{v_{2}}{\tilde{x}_{2}}+\left(1-E_{s}\right) \hat{x}_{3}
$$

Provided that the system trajectories evolve outside the manifold of singularity $\mathcal{S}$, the output of this observer provides a finite time estimation of $x$ and $m$ :

Step 1: From the section 2.2, there exists a time $t_{1}>0$ such that, $\forall t>t_{1}$, the first exact differentiator gives $\hat{x}_{1}=x_{1}$ and $\tilde{x}_{2}=x_{2}$

Step 2: For $t>t_{1}, E_{1}=1$. Thus $\exists t_{2}>t_{1}$ such that $\forall t>t_{2}$, the second exact differentiator gives $\hat{x}_{2}=\tilde{x}_{2}=x_{2}$ and $v_{2}=x_{2} x_{3}$. If $\left|x_{2}\right|>\varepsilon$, then $E_{s}=1$ and the relation (13) gives $\tilde{x}_{3}=x_{3}$. Otherwise, $E_{s}=0$ and $\tilde{x}_{3}=\hat{x}_{3}$.

Step 3: For $t>t_{2}, E_{2}=1$. If $E_{s}=1$, the observation error dynamics of $e_{3}=x_{3}-\hat{x}_{3}$ is equal to:

$$
\dot{e}_{3}=m-K \operatorname{sign}\left(e_{3}\right)
$$

and, for sufficiently large $K$, a first order sliding motion appears on $e_{3}=0$. The equivalent dynamics provides an estimation of the unknown input $m$. Let us now assume that the system is on $\mathcal{S}$. In that case, $E_{s}=0$ and

$$
\begin{aligned}
\dot{e}_{3} & =m+e_{3}-\left(1+x_{1}^{2}+x_{2}^{2}\right)\left(x_{3}^{3}-\hat{x}_{3}^{3}\right) \\
& =m+e_{3}\left[1-\left(1+x_{1}^{2}+x_{2}^{2}\right)\left(x_{3}^{2}+\hat{x}_{3} x_{3}+\hat{x}_{3}^{2}\right)\right]
\end{aligned}
$$

\footnotetext{
${ }^{3}$ but also nonlinear observers based on other methodologies.
} 
Thus, the observer can be viewed as an "estimator". The system (15) can be shown to be stable for sufficiently large $\hat{x}_{3}$ and, consequently, $e_{3}$ remains always bounded.

Since the manifold of observability bifurcation $\mathcal{S}$ is not an invariant set except for the trivial solution $m=x_{1}=x_{2}=0$ (or very particular $m$ ), the observer will converge in finite time to $e_{3}=0$. Note that for $m=x_{2}=x_{1}=0$, both the state $x_{3}$ and the observed state $\hat{x}_{3}$ asymptotically converge to 1 .

This type of observability singularity have to be overcome in applications such as bioprocess [51] or induction motor without mechanical sensor [10]. On another hand, it can be very useful for particular application such as secure data transmission [3]. Indeed, the confidential message can be hidden in some observability singularity and one has to design a variable structure observer to recover it.

\subsection{Switched system}

A particular model of interest in hybrid systems are continuous-time systems with discrete switching events from a certain class. Such systems are called switched systems and there is an abundant literature on the observation problem (see e.g. $[1,5,14,61]$ and the references therein). Let us consider the following nonlinear switched system without jump constituted, for a sake of simplicity, of two nonlinear subsystems:

$$
\left\{\begin{array}{l}
\dot{x}=f_{1}(x) \text { and } y=h_{1}(x) \text { if } \sigma(x) \leq 0 \\
\dot{x}=f_{2}(x) \text { and } y=h_{2}(x) \text { if } \sigma(x)>0
\end{array}\right.
$$

where $x \in \mathcal{R}^{n}, y \in \mathcal{R}$ is the output, $f_{i}(x)$ are smooth vector fields, and $h_{i}(x)$, $\sigma(x)$ are smooth functions. The continuous state space $\mathcal{R}^{n}$ is partitioned into two operating regions by means of the switching surface $\sigma(x)=0$. Let us denote the switching times $t_{1}, t_{2}, \ldots$ A difficulty arises from the fact that the observer has to converge in a time less than the evolution duration $t_{i+1}-t_{i}$ (before each switching of the system). Here, it is shown that the use of the step-by-step sliding mode observer can be of high interest for the observation problem of switched systems.

It is assumed that:

A.1 the system (16) is bounded state in finite time ${ }^{4}$ and without jump.

A.2 each subsystem is observable i.e. for $i=1: 2$,

$$
\operatorname{rank}\left\{d h_{i}, d L_{f_{i}} h_{i}, \ldots, d L_{f_{i}}^{n-1} h_{i}\right\}=n .
$$

A.3 $t_{i+1}-t_{i}>\delta$ for all $i . \delta$ is called the dwell time [44].

\footnotetext{
${ }^{4}$ The assumption of bounded state must concern the whole system. Indeed, all the subsystems can be perfectly stable, while the global system can be unstable due to the switching phenomenon.
} 
Assumption A.3 means that systems with Zeno phenomenon are not considered. Under those assumptions, one can conclude that the global system (16) is observable if the dynamics of evolution of the system is known. Let us consider the state transformation defined by:

$$
z_{i+1}^{j}=\phi_{i+1}^{j}(x)=L_{f_{j}}^{i} h_{j}(x) \quad \text { for } 0 \leq i \leq n-1, j=1: 2 .
$$

In these local coordinate charts, each subsystem of (16) can be written in a triangular observable form:

$$
\left\{\begin{array}{l}
\dot{z}_{i}^{1}=z_{i+1}^{1} \quad \text { for } i=1: n-1 \\
\dot{z}_{n}^{1}=g_{1}\left(z_{1}^{1}, z_{2}^{1}, \ldots, z_{n}^{1}\right)
\end{array}\right.
$$

if $\sigma_{1}:=\left(\phi^{1}\right)^{-1}\left(z_{1}^{1}, z_{2}^{1}, \ldots, z_{n}^{1}\right) \leq 0$, and

$$
\left\{\begin{array}{l}
\dot{z}_{i}^{2}=z_{i+1}^{2} \quad \text { for } i=1: n-1 \\
\dot{z}_{n}^{2}=g_{2}\left(z_{1}^{2}, z_{2}^{2}, \ldots, z_{n}^{2}\right)
\end{array}\right.
$$

if $\sigma_{2}:=\left(\phi^{2}\right)^{-1}\left(z_{1}^{2}, z_{2}^{2}, \ldots, z_{n}^{2}\right)>0$.

Remark 4 The assumption of a system without jump is necessary because the diffeomorphism $\phi^{1}$ linked to the system $i=1$ is not generally the same than the diffeomorphism $\phi^{2}$ linked to the system $i=2$. Then at each switch, the state in triangular observable form may jump even if the state in the original coordinates does not jump. Jumps can be taken into account by a new structure of sliding mode observer that will be given in a forthcoming paper. Nevertheless, in the case of switched Lagrangian systems, the considered assumption is satisfied [53]. Note also that when the diffeomorphisms $\phi^{i}$ are the same (again, it is the case for switched Lagrangian systems), the step-by-step sliding mode observer is a common observer to all subsystems and does not require the knowledge of the switching function $\sigma$.

The finite time sliding mode observer (5) is of interest for the two main following reasons:

1. Under assumptions A.1 and A.2, it can provide an estimation $\tilde{z}_{i}^{j}$ of the state in a finite time $T_{f}$. Thus, if the observer gains can be chosen such that $T_{f}<\delta$, one has the knowledge of the state between each switching of the system.

A lot of observers for switched systems in the literature assume the knowledge of the operating regions (i.e. the value of the switching function $\sigma$ ). Here, under the assumption that $g_{i}(x)$ and $\dot{g}_{i}(x)$ are bounded, one can get the continuous equivalent output injection (in the $n$-th step of the convergence of the observer) $\tilde{\theta}=g_{j}\left(z_{1}^{j}, z_{2}^{j}, \ldots, z_{n}^{j}\right)$ that can be compared to each value of $g_{j}\left(\tilde{z}_{1}^{j}, \tilde{z}_{2}^{j}, \ldots, \tilde{z}_{n}^{j}\right), j=1,2$ in order to know the operating region where the system evolves (see [7] or [11] for more details on the geometrical conditions establishing this region). 
2. In [16], a step-by-step first order sliding mode observer was mainly employed for the same reasons: the finite time convergence and the ability to take naturally into account the variable structure of the hybrid system. Nevertheless, some difficulties occurred due to the chattering phenomena. It induced some irrelevant decision of switching between the subsystems when the trajectory was in the neighborhood of the switching manifold. This problem was bypassed by using a low pass filter during the computation of the equivalent vector. However, this solution introduced a delay in the observed state (asymptotic convergence) and again that could lead to false information about the discrete state of the system. Thus, the second order sliding mode observer can be a powerful and efficient tool to solve this problem of observation because of its ability to provide a continuous and accurate finite time estimation.

Further work may deal with the design of observers for switched or impact systems with jumps and non autonomous systems which turns out to be particularly useful in many applications.

\subsection{Extension of the matching condition for nonlinear sys- tems}

In [24], the authors presented an observation algorithm in order to put a linear system with unknown inputs in a set of block triangular observable forms, even if the matching condition was not fulfilled, and a finite time sliding mode observer was designed in order to estimate the state and the unknown inputs in [25]. The main idea of this algorithm is to take advantage of some equivalent output injections in order to generate fictitious outputs such that the system can be transformed into a set of block observable triangular forms. The first block of the triangular form is fed by the original inputs of the system while the subsequent blocks are fed by the fictitious outputs. Here, it is shown via an example that this idea can also be applied for some class of nonlinear systems that can not be put in the form (2)-(3). Let us consider the following nonlinear system:

$$
\begin{aligned}
\left(\begin{array}{l}
\dot{x}_{1} \\
\dot{x}_{2} \\
\dot{x}_{3} \\
\dot{x}_{4}
\end{array}\right)=\left(\begin{array}{c}
x_{2} \\
\sin \left(x_{1}\right)-x_{4}+m_{1} \\
x_{4}+m_{1} \\
\cos \left(x_{3}\right)+m_{2}
\end{array}\right) \\
\left(\begin{array}{l}
y_{1} \\
y_{2}
\end{array}\right)=\left(\begin{array}{l}
h_{1} \\
h_{2}
\end{array}\right)=\left(\begin{array}{l}
x_{1} \\
x_{3}
\end{array}\right)
\end{aligned}
$$

where all the states are bounded $\forall t \geq 0$ and where $m_{1}, m_{2}$ are some bounded unknown inputs. Figure 1 is a block diagram of (19)-(20).

For this system, one has $\rho_{1}=2$ and $\rho_{2}=1$ with respect to the outputs $y_{1}$ and $y_{2}$. Thus, according to Proposition 1, (19)-(20) can not be transformed into a form similar to (2)-(3), and as a consequence usual sliding mode observers fail 


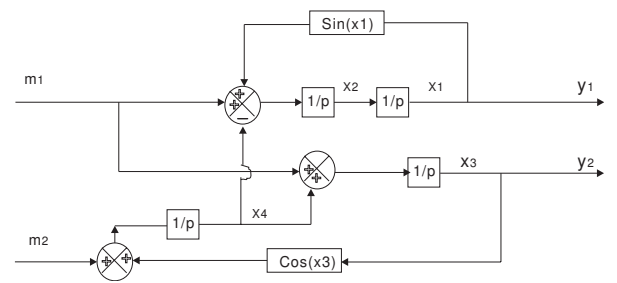

Figure 1: System representation of (19)-(20)

to estimate the state in finite time. Furthermore, the matrix

$$
\Gamma(x)=\left(\begin{array}{ll}
L_{g_{1}} L_{f}^{\rho_{1}-1} h_{1}(x) & L_{g_{2}} L_{f}^{\rho_{1}-1} h_{1}(x) \\
L_{g_{1}} L_{f}^{\rho_{2}-1} h_{2}(x) & L_{g_{2}} L_{f}^{\rho_{2}-1} h_{2}(x)
\end{array}\right)=\left(\begin{array}{ll}
1 & 0 \\
1 & 0
\end{array}\right)
$$

is singular $\forall x \in \mathcal{R}^{4}$ and the method developed in [63], that could eventually lead to the asymptotic estimation of $x$, can neither be applied.

Let us show that one can even though estimate both state and unknown inputs using the finite time sliding mode observer described in Section 2.2. Define a fictitious output as $y_{3}=y_{2}-\dot{y}_{1}=x_{3}-x_{2}$. One has $\rho_{1}=2, \rho_{2}=1$ and $\rho_{3}=2$ with respect to the fictitious output $\bar{y}=\left[y_{1}, y_{2}, y_{3}\right]^{T}$, while the observability indices are $\{1,1,2\}$. The change of coordinates $\phi(x)=\left[\xi_{1}, \xi_{2}, \xi_{3}, \xi_{4}\right]^{T}=$ $\left[y_{1}, y_{2}, y_{3}, x_{4}\right]^{T}=\left[x_{1}, x_{3}, x_{3}-x_{2}, x_{4}\right]^{T}$, defined for all $x \in \mathcal{R}^{4}$, transforms the system into a set of block triangular observable forms with respect to $\bar{y}$ :

$$
\left(\begin{array}{c}
\dot{\xi}_{1} \\
\dot{\xi}_{2} \\
\dot{\xi}_{3} \\
\dot{\xi}_{4}
\end{array}\right)=\left(\begin{array}{c}
\xi_{2}-\xi_{3} \\
\xi_{4}+m_{1} \\
-\sin \left(\xi_{1}\right)+2 \xi_{4} \\
\cos \left(\xi_{2}\right)+m_{2}
\end{array}\right)
$$

Figure 2 is a representation scheme of (21).

Following the lines of Section 2.2, a state observer can be designed as:

$$
\left\{\begin{array}{l}
\dot{\hat{x}}_{1}=\tilde{x}_{2}+\lambda_{1}\left|\tilde{e}_{1}\right|^{1 / 2} \operatorname{sign}\left(\tilde{e}_{1}\right) \\
\dot{\tilde{x}}_{2}=\alpha_{1} \operatorname{sign}\left(\tilde{e}_{1}\right) \\
\dot{\hat{\xi}}_{3}=E_{1}\left[-\sin \left(y_{1}\right)+2 \tilde{\xi}_{4}+2 \lambda_{3}\left|\tilde{e}_{3}\right|^{1 / 2} \operatorname{sign}\left(\tilde{e}_{3}\right)\right] \\
\dot{\tilde{\xi}}_{4}=E_{1} \alpha_{3} \operatorname{sign}\left(\tilde{e}_{3}\right)
\end{array}\right.
$$

where $\tilde{e}_{1}=y_{1}-\hat{x}_{1}, \tilde{e}_{3}=\xi_{3}-\hat{\xi}_{3}$ and with:

$$
E_{1}=1 \text { if }\left|\tilde{e}_{1}\right|=\left|y_{1}-\hat{x}_{1}\right| \leq \varepsilon, \text { else } E_{1}=0 .
$$

It can be seen that (22) is made of two exact differentiators.

Step 1: $\exists t_{1}>0$ such that $\forall t>t_{1}$ the first exact output differentiator provides 


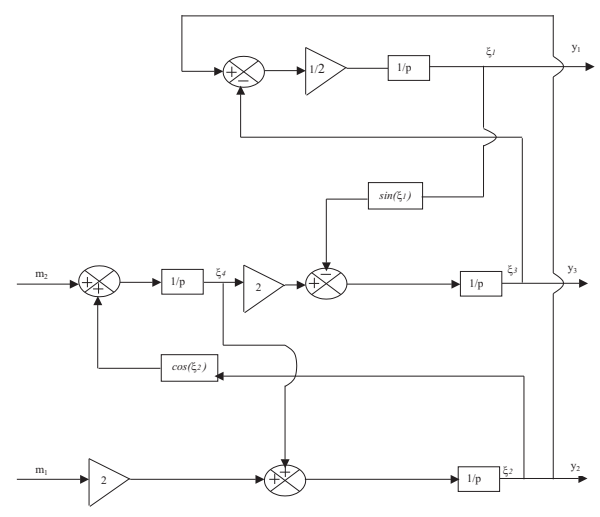

Figure 2: System representation of (21)

$\hat{x}_{1}=x_{1}, \tilde{x}_{2}=x_{2}$ and $E_{1}=1$. The previously introduced fictitious output $y_{3}=\xi_{3}=y_{2}-\tilde{x}_{2}$ is henceforth available.

Step 2: Similarly to the first step, $\exists t_{2}>t_{1}$ such that $\forall t>t_{2}$, the second exact differentiator gives $\hat{\xi}_{3}=\xi_{3}$ and $\tilde{\xi}_{4}=\xi_{4}$. Thus, the full original state $\left[x_{1}, x_{2}, x_{3}, x_{4}\right]^{T}=\left[y_{1}, y_{2}-\xi_{3}, y_{2}, \xi_{4}\right]^{T}$ has been obtained in finite time.

Now, let us assume that $\dot{m}_{1}, \dot{m}_{2}$ are bounded. The following observer provides a finite time estimation of $m_{1}$ and $m_{2}$ :

$$
\left\{\begin{array}{l}
\dot{\hat{\xi}}_{2}=E_{o b s}\left[\tilde{x}_{4}+\tilde{m}_{1}+\lambda_{m_{1}}\left|\tilde{e}_{2}\right|^{1 / 2} \operatorname{sign}\left(\tilde{e}_{2}\right)\right] \\
\dot{\tilde{m}}_{1}=E_{o b s} \alpha_{m_{1}} \operatorname{sign}\left(\tilde{e}_{2}\right) \\
\dot{\hat{\xi}}_{4}=E_{o b s}\left[\cos \left(y_{2}\right)+\tilde{m}_{2}+\lambda_{m_{2}}\left|\tilde{e}_{4}\right|^{1 / 2} \operatorname{sign}\left(\tilde{e}_{4}\right)\right] \\
\dot{\tilde{m}}_{2}=E_{o b s} \alpha_{m_{2}} \operatorname{sign}\left(\tilde{e}_{4}\right)
\end{array}\right.
$$

where

$$
\begin{aligned}
E_{o b s} & =0 \text { if } t<t_{2}, \\
\text { else } E_{o b s} & =1 .
\end{aligned}
$$

For $t>t_{2}$, the first part of the observer has converged, all the state is available and $E_{\text {obs }}=1$. Then, the output of the two exact differentiators in (23) gives a finite time estimation of $\tilde{m}_{1}=m_{1}$ and $\tilde{m}_{2}=m_{2}$.

The observer design can be represented by the scheme given in Figure 3 .

Remark 5 The finite time sliding mode observers designed in [43] and in [6] can be also used in this kind of problem. 


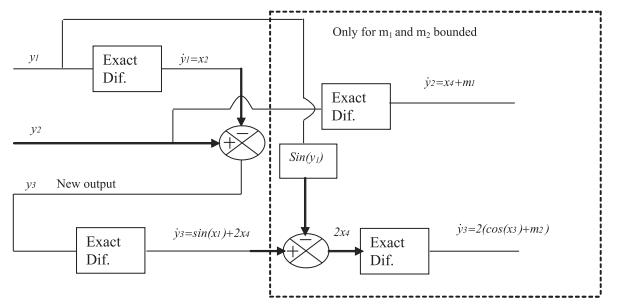

Figure 3: Observer scheme

\section{Conclusion}

In this paper, we considered the problem of finite time estimation of MIMO nonlinear systems with unknown inputs. Structural conditions stating on the possibility to design sliding mode observers for finite time estimation of both state and unknown inputs have been derived. Then, a finite time sliding mode observer based on the iterative use of the super twisting algorithm has been proposed. It was shown that this kind of observer could be a powerful tool to solve quite difficult problems of observation, like the design of observers for switched systems. A modified version of the sliding mode observer was designed for systems with observability singularities. It should be stressed that few papers are devoted to the problem of sliding mode observer design for these two promising fields of investigation. Lastly, by the way of an illustrative example, a finite time observer was designed for a nonlinear system that do not satisfies the matching condition. Further works aim at generalizing this idea.

\section{References}

[1] A. Balluchi, L. Benvenuti, M. D. Di Benedetto and A.L. SangiovanniVincentelly, "Design of Observer for Hybrid Systems", in: Lecture notes in computer Science 2289, C. J. Tomlin and M. R. Greenstreet, Eds, Springer Verlag, pp. 77-89, 2002.

[2] J.-P. Barbot, T. Boukhobza and M. Djemaï, "Sliding mode observer for triangular input form", IEEE Conference on Decision and Control, Kobe, Japan, 1996.

[3] J.-P. Barbot, I. Belmouhoub and L. Boutat-Baddas, "Observability Normal Forms", in: New trends in Nonlinear dynamics and control, LNCIS 295, W. Kang et al., Eds, Springer Verlag, pp. 1-24, 2003.

[4] G. Bartolini, A. Pisano and E. Usai, "First and second derivative estimation by sliding mode technique", Journal of Signal Processing, 4, pp. 167-176, 2000 . 
[5] A. Bemporad, G. Ferrari-Trecate and M. Morari, "Observability and controllability of piecewise affine and hybrid systems", IEEE Transactions on Automatic Control, 45, pp. 1864-1876, 2000.

[6] A. Bejarano, F. Poznyak and L. Fridman "Observer for linear time invariant systems with unknown inputs based on the hierarchical super-twisting concept", International Workshop on Variable Structure Systems, Alghero, Sardinia, 2006.

[7] D. Boutat, A. Benali and J.-P. Barbot, "About the observability of piecewise dynamical systems", NOLCOS, Stuttgart, Germany, 2004.

[8] K. Busawon and M. Saif, "A state observer for nonlinear systems", IEEE Transactions on Automatic Control, 44, pp. 2098-2103, 1999.

[9] C. Canudas de Wit and J. J. E. Slotine, "Sliding observers in robot manipulators", Automatica, 27, pp. 859-864, 1991.

[10] C. Canudas De Wit, A.Youssef, J.-P. Barbot, Ph. Martin and F. Malrait, "Observability conditions of induction motors at low frequencies", IEEE Conference on Decision and Control, Sydney, Australia, 2000.

[11] S. Chaib, D. Boutat, A. Benali and J.-P. Barbot, "Observability of the discrete state for dynamical piecewise hybrid systems", Nonlinear Analysis: Theory, Methods 83 Applications, 63, pp 423-438, 2005.

[12] M. Darouach, M. Zasadzinski and S. J. Xu, "Full-order observers for linear systems with unknown inputs", IEEE Transactions on Automatic Control, 39, pp. 606-609, 1994.

[13] J. Davila, L. Fridman and A. Levant, "Second-order sliding-mode observer for mechanical systems", IEEE Transactions on Automatic Control, 50, pp. 1785-1790, 2005.

[14] M. De la Sen and N. Luo, "Design of linear observers for a class of linear hybrid systems", Int. Journal of Systems Science, 31, pp. 1077-1090, 2000.

[15] M. Djemaï, J.-P. Barbot, A. Glumineau and R. Boisliveau, "Nonlinear flux sliding mode observer", Proceedings of the IEEE CSCC99, IMACS, Athens, Greece, 1999.

[16] M. Djemaï, N. Manamani and J. P. Barbot, "Sliding mode observer for triangular input hybrid system", IFAC World congress, Praha, 2005.

[17] B. Drazenovic, "The invariance conditions in variable structure systems", Automatica, 5, pp. 287-295, 1969.

[18] S. V. Drakunov, "Sliding-mode Observer Based on Equivalent Control Method", IEEE Conference on Decision and Control, Tucson, Arizona, 1992 . 
[19] S. V. Drakunov and V. I. Utkin, "Sliding mode observers. Tutorial", IEEE Conference on Decision and Control, New-Orleans, LA , 1995.

[20] C. Edwards and S. K. Spurgeon, "On the development of discontinuous observers", International Journal of control, 59, pp. 1211-1229, 1994.

[21] C. Edwards and S. K. Spurgeon, Sliding mode control: theory and applications, Taylor and Francis, Eds, 1998.

[22] C. Edwards and S. K. Spurgeon and R. J. Patton, "Sliding mode observers for fault detection and isolation", Automatica, 36, pp. 541-553, 2000.

[23] T. Floquet, J.-P. Barbot, W. Perruquetti and M. Djemaï, "On the robust fault detection via a sliding mode disturbance observer", International Journal of control, 77, pp. 622-629, 2004.

[24] T. Floquet and J.-P. Barbot, "An observability form for linear systems with unknown inputs", International Journal of control, 79, pp. 132-139, 2006a.

[25] T. Floquet and J.-P. Barbot, "A canonical form for the design of unknown input sliding mode observers", in: Advances Variable Structure and Sliding Mode Control, Lecture Notes in Control and Information Sciences, Vol. 334, C. Edwards, E. Fossas Colet, L. Fridman, Eds., Springer Edition, 2006b.

[26] F. Floret-Pontet and F. Lamnabhi-Lagarrigue, "Parametric identification methodology using sliding modes observer", International Journal of Control, 74, pp. 1743-1753, 2001.

[27] R. Garrido, Y. B. Shtessel, L. M. Fridman, "Application of VS differentiators to DC servomechanisms", International Workshop on Variable Structure Systems, Barcelona, Spain, 2004.

[28] J-P. Gauthier and G. Bornard, "Observability for any $u(t)$ of a class of bilinear systems", IEEE Transactions on Automatic Control, 26, No 6, pp. 922-926, 1981.

[29] J. P. Gauthier, H. Hammouri and S. Othman, "A simple observer for nonlinear systems with applications to bioreactors", IEEE Transactions on Automatic Control, 37, pp. 875-880, 1992.

[30] K. B. Goh, S. K. Spurgeon, N. B. Jones, "Fault diagnostics using sliding mode techniques", Control Engineering Practice, 10, pp. 207-217, 2002.

[31] Y. Guan and M. Saif, "A novel approach to the design of unknown input observers", IEEE Transactions on Automatic Control, 36, pp. 728-740, 1991.

[32] I. Haskara, Ü. Özgüner and V. I. Utkin, "On sliding mode observers via equivalent control approach", International Journal of control, 71, pp. 1051-1067, 1998. 
[33] I. Haskara and Ü. Özgüner, "Equivalent value filters in disturbance estimation and state observation", in: Lecture Notes in Control and Information Science, Vol. 24\%, pp. 167-179, 1999.

[34] R. Hermann and A. J. Krener, "Nonlinear controllability and observability", IEEE Transactions on Automatic Control, 22, pp. 728-740, 1977.

[35] J. Hernandez and J-P. Barbot, "Sliding observer-based feedback control for flexible joints manipulator", Automatica, 32, pp. 1243-1254, 1996.

[36] M. Hou and P. C. Müller, "Design of observers for linear systems with unknown inputs", IEEE Transactions on Automatic Control, 37, pp. 871$875,1992$.

[37] M. Hou, K. Busawon, and M. Saif, "Observer design for nonlinear systems via injective mapping", IEEE Transactions on Automatic Control, 45, pp. 1350-1355, 2000.

[38] A. Isidori, Nonlinear Control Systems, Communication and Control Engineering Series, Third edition, Springer-Verlag, 1995.

[39] A. J. Krener and W. Respondek, "Nonlinear observers with linearizable error dynamics", SIAM J. Control Optim., 23, pp. 197-216, 1985.

[40] A. Levant, "Sliding order and sliding accuracy in sliding mode control", International Journal of Control, 58, pp. 1247-1263, 1993.

[41] A. Levant, "Robust exact differentiation via sliding mode technique", $A u$ tomatica, 34, pp. 379-384, 1998.

[42] A. Levant, "Higher-order sliding modes, differentiation and outputfeedback control", International Journal of Control, 76, pp. 924-941, 2003.

[43] A. Levant, "Homogeneity approach to high-order sliding mode design", Automatica, 41, pp. 823-830, 2005.

[44] D. Liberzon, Switching in systems and control, Birkhäuser, 2003.

[45] N. Manamanni, M. Djemaï, T. Boukhobza and N. M'Sirdi, "Nonlinear sliding observer based control for a pneumatic robot leg", Int. J. of Robotics and Automation, 16, pp.100-112, 2001.

[46] R. Martinez-Guerra, R. Aguilar-Lopez, A. Poznyak, "Robust sliding-mode observers for on-line reaction heat monitoring in continuous chemical reactors", ASME Journal of Dynamics Systems, Measurement \& Control, 126, pp. 473-478, 2004.

[47] Y. Orlov, L. Aguilar, and J. C. Cadiou, "Switched chattering control vs. backlash/friction phenomena in electrical servo-motors," International Journal of Control, 76, pp. 959-967, 2003. 
[48] W. Perruquetti and J.-P. Barbot, Sliding Mode Control in Engineering, Ed. Marcel Dekker, 2002.

[49] A. Pisano and E. Usai, "Output-feedback control of an underwater vehicle prototype by higher-order sliding modes," Automatica, 40, pp. 1525-1531, 2004.

[50] A. S. Poznyak, "Stochastic output noise effects in sliding mode estimations", International Journal of Control, 76, pp. 986-999, 2003.

[51] A. Rapaport and A. Maloum, "Embedding for exponential observers of nonlinear systems", IEEE Conference on Decision and Control, Sydney, Australia, 2000.

[52] J. Reger, H. Sira-Ramirez and M. Fliess, "On non-asymptotic observation of non-linear systems", IEEE Conference on Decision and Control, Sevilla, 2005.

[53] H. Saadaoui, N. Manamanni, M. Djemaï, J.P. Barbot and T. Floquet, "Exact differentiation and sliding mode observers for switched Lagrangian systems", Nonlinear Analysis: Theory, Methods 83 Applications, 65, pp. 1050-1069, 2006.

[54] Y. B. Shtessel, I. A. Shkolnikov, and M. D. J. Brown, "An asymptotic second-order smooth sliding mode control," Asian Journal Control, 5, pp. 498-504, 2003.

[55] Y. B. Shtessel and A. S. Poznyak, "Parameter identification of linear time varying systems via traditional and high order sliding modes", International Workshop on Variable Structure Systems, Barcelona, Spain, 2004.

[56] J. J. Slotine, J. K. Hedrick, and E. A. Misawa, "On sliding observers for nonlinear systems", ASME J. Dyn. System Measurement Control, 109, pp. $245-252,1987$.

[57] C. P. Tan and C. Edwards, "An LMI approach for designing sliding mode observers", International Journal of Control, 74, pp. 1559-1568, 2001.

[58] C. P. Tan and C. Edwards, "Sliding mode observers for robust detection and reconstruction of actuator and sensor faults", Int. J. of robust and nonlinear control, 13, pp. 443-463, 2003.

[59] V. I. Utkin, Sliding Modes in Control and Optimization, Berlin, Germany, Springer-Verlag, 1992.

[60] V. I. Utkin, J. Guldner and J. Shi, Sliding mode control in electromechanical systems, Taylor and Francis, London, 1999.

[61] R. Vidal, A. Chiuso, S. Soatto and S. S. Sastry, "Observability of linear hybrid systems, in: Hybrid Systems: Computation and Control, Lecture Notes in Computer Science, Springer Verlag, Vol. 2623, pp. 526-539, 2003. 
[62] B. L. Walcott and S. H. Żak, "State observation of nonlinear uncertain dynamical systems", IEEE Transactions on Automatic Control, 32, pp. 88-104, 1987.

[63] Y. Xiong and M. Saif, "Sliding mode observer for nonlinear uncertain systems", IEEE Transactions on Automatic Control, 46, pp. 2012-2017, 2001. 